\title{
An Integrated Optimization System for Safe Job Assignment Based on Human Factors and Behavior
}

\author{
Beatrice Lazzerini, Member, IEEE, and Francesco Pistolesi
}

\begin{abstract}
Industrial safety has been deeply improved in the last years thanks to increasingly sophisticated technologies. Nevertheless, 2.3 million people yearly die worldwide due to occupational illnesses and accidents at work. Human factors can be profitably used for safety improvement because of their influence on the workers' behavior. This paper presents an integrated optimization system to help companies assign each task to the most suitable worker, minimizing cost, while maximizing expertise and safety. The system is made of three modules. A neural module computes each worker's caution for every task on the basis of some human factors and the worker's behavior. To solve the multi-objective job assignment problem, an evolutionary module approximates the Pareto front through the NSGA-II algorithm. Pareto-optimal solutions then form the alternatives of a multi-criteria decision making problem, and the best is selected by a decision module jointly based on the Analytic Hierarchy Process (AHP) and the Technique for Order of Preference by Similarity to Ideal Solution (TOPSIS). Validation was carried out involving two footwear companies where personnel was recruited and reassigned to tasks, respectively. Comparing the worker-task assignment proposed by the system to the one suggested/used by the management, noteworthy low-cost improvement in safety is shown in both scenarios, with low or no decrease in expertise. The proposed system can thus contribute to get safer workplaces where risks are less likely and/or less harmful.
\end{abstract}

Index Terms-Decision making, human factors, occupational safety, optimization, risk analysis.

\section{INTRODUCTION}

$\mathbf{S}$ AFETY is paramount every time a job is assigned to a person. There were just under 2.5 million and 3,515 nonfatal and fatal accidents at work, respectively, in the EU in 2012 [1]. In the USA, over the last 5 years, net increases to the preliminary count of fatal injuries have averaged 173 cases, ranging from 84 in 2011 (up 2\%) to 245 in 2012 (up 6\%). Fatal injuries were higher in manufacturing (up 9\%), mining (up 17\%), construction (up 6\%) and agriculture (up 14\%) [2]. Worldwide, 4 people die every minute as a consequence of occupational illnesses and accidents at work [3]. Physical and chemical hazards are typically the most important cause of occupational injuries in manufacturing industries [3]. In several of these, physical hazards are perhaps unavoidable. Serious injuries and fatalities are often caused by machines, typically made of moving or rotating parts, sharp edges and extremely hot surfaces. Machines can crush, stab, cut, shear, burn, wound or strike workers, if used unsafely. Extreme temperatures can also be dangerous. Heat stress

Manuscript received...

B. Lazzerini and F. Pistolesi are with the Department of Information Engineering, University of Pisa, Largo Lucio Lazzarino 1, 56122 Pisa (IT).

This research was supported by the PRA 2016 project "Analysis of Sensory Data: from Traditional Sensors to Social Sensors", funded by the University of Pisa. potentially causes cramps, exhaustion, heat stroke and rashes; overexposure to cold can cause hypothermia and frostbite. Electricity also poses a danger to workers. Fatal electrocution, electric shock, burns, and falls caused by contact with electric energy are typical electrical injuries.

On the other side, chemical hazards are concerned with the consequences of solvents, acids, bases, particulates and many others on human health. Consequences include disfiguring burns, blindness caused by corrosive chemicals and poisoning.

Zero harm is wished for next-generation workplaces to remove all negative effects of work on health. With the huge amount of occupational accidents discussed, all recent improvements in safety are far from guaranteeing safe workplaces. Maybe the workers' behavior in dealing with risk should be much more deeply taken into account, to avoid dangerous actions which can trigger devastating events.

In the last decades, because of the ever-increasing reliability of machines and technical systems, the industrial safety focus has turned to human causes of accidents. It is estimated that up to $80 \%$ of the accidents may stem from people's actions or omissions, at least in part [4]. In particular, many accidents are blamed on actions or omissions of individuals while being involved in operational or maintenance work.

Workers' actions are influenced by humans factors, which refer to environmental, organizational and job factors, and to individual characteristics which influence the behavior at work in a way that can impact on health and safety [4]. Human factors thus affect risk perception, i.e., the way one estimates characteristics and gravity of dangerous situations [5]-[9]. With reference to the individual aspect, people are characterized by their own skills, personal attitudes, habits and personalities, which can represent strengths or weaknesses, depending on the task requirements. Individual characteristics influence behavior in complex ways. Some characteristics, such as personality, are essentially fixed and they cannot be changed easily. The others, such as attitudes and skills, may be enhanced. By considering human factors when dealing with industrial safety, the number of accidents and cases of occupational illnesses can be reduced [4]. Some human factors used in this paper are past experience and knowledge, past health status (e.g., diseases and accidents at work), psychological (e.g., anxiety), social, political, and cultural factors, mood and emotions, trust in risk management institutions, age, personal knowledge about risks, locus of control [10], [11], optimism bias [11], [12]. These factors have been studied thoroughly, but nowadays there is no exhaustive explanation of why a person behaves this or that way in the presence of risk.

Specific risk programs promote risk-aware culture in companies [13], letting workers acquire knowledge on how they 
can safely deal with risks every day. Risk programs include risk awareness training, which is generally iterated to stress the concepts so that learning is reinforced, with the aim of an ever-increasing injury reduction. This is quite expensive for companies. The training outcome can also be enhanced by using training methods specifically tailored to every single worker [14], exploiting his/her sensitivity to risk [15]. Anyway, significant economic resources are required.

In the EU, about $99 \%$ of the companies are small and medium enterprises (SMEs), with low number of employees. SMEs employ 65 million people altogether [16]. As an example, outstanding manufacturing excellence and small size are fairly common for the Italian industry, widely made of family SMEs, where keeping costs as low as possible is crucial. Workrelated injuries and illnesses cause costs ranging from $1.8 \%$ to $6 \%$ of GDP, with an average of $4 \%$ [3]. Risk awareness training generally requires sophisticated tools for risk analysis and management [17], [18], which cause unaffordable costs for SMEs. Moreover, in spite of safety programs, occupational accidents and fatalities repeatedly recur with the numbers discussed so far. That is why it may be helpful for human factors and behavior to be assessed every time a task is assigned to a person. An approach like this could make hazards less likely as well as less harmful. Job assignment problems classically take cost into account, hence they should include at least two objectives: cost and safety. Job assignment is therefore a multi-objective optimization problem (MOO).

This paper presents an integrated optimization system which helps the management of a company assign the most suitable person to each task. The system is based on a novel multiobjective formulation of the job assignment problem including three objectives, namely, economic cost, workers' expertise, and safety. The formulation proposed aims to obtain affordable worker-task assignments where each worker is enough experienced and careful with respect to the task assigned. The system is composed of three modules, described in the following.

The neural module determines each worker's risk perception and caution levels with respect to the risks of every single task, taking as inputs the worker's values for the human factors and his/her behavior while executing the task. The worker's behavior is represented by means of one or more preventive actions that the worker performs or would perform while executing the task. Each worker's level of safety in performing every task is determined by aggregating his/her risk perception and caution levels towards that task.

For each pair (worker,task), the evolutionary module receives: i) the worker's level of safety in executing the task; ii) the cost of assigning the worker to the task; iii) the worker's experience for the task. These measures, together with the identifiers of the safety-critical tasks, are used by the evolutionary module to generate near Pareto-optimal personnel assignments through a customized version of the NSGA-II algorithm, which maintains feasibility during the evolution. In particular, swap mutation and partially matched crossover are used, so as to handle constraints by repairing infeasible solutions as they are generated.

To select the best solution, a hybrid multi-criteria decision making (MCDM) technique is implemented by the decision module, which considers each assignment generated by the evolutionary module as an alternative of an MCDM problem. It then selects the best solution with the Technique for Order of Preference by Similarity to Ideal Solution (TOPSIS), using the decision maker's preferences for the objectives, derived with the Analytic Hierarchy Process (AHP).

The paper is organized as follows: Section II contains some preliminaries on multi-criteria decision making and multiobjective optimization; Section III presents the model of the worker's risk perception and caution used by the neural module; Section IV gives the problem formulation and the objective functions used by the evolutionary module; Section V contains a detailed description of the system; in Sections VI and VII the experiments are described and discussed, respectively. Section VIII draws the conclusions.

\section{PRELIMINARIES}

\section{A. Multi-criteria decision making}

Multi-criteria decision making (MCDM) deals with decision problems having a goal, a set of criteria and a set of alternatives. Criteria and alternatives form the elements of the MCDM problem. The goal is to find the best alternative with respect to all the criteria.

1) Analytic Hierarchy Process (AHP): AHP is an MCDM method that structures elements as a hierarchy [19]. In the simplest case, a three-level hierarchy is built, containing goal, criteria and alternatives, from top to bottom. Each criterion is connected to the goal and to all alternatives.

An $n \times n$ pairwise comparison matrix (hereafter referred to as PCM) $\mathbf{P}=\left[p_{i j}\right]$ is built for each level of the hierarchy to compare elements of that level to each other, with respect to each shared parent, where $n$ is the number of elements in the level, and $i, j \in\{1, \ldots, n\}$. It holds that $p_{i j}>0, p_{i j}=1 / p_{j i}$, and $p_{i i}=1 \forall i, j \in\{1, \ldots, n\}$. Entries $p_{i j}$, called preferences, estimate the preference of $i$ over $j$, and are typically expressed using the Saaty's scale (see Table I). In this case, $\mathbf{P}$ is a

TABLE I

SAATY'S SCALE OF PREFERENCE

\begin{tabular}{cl}
\hline Preference & Explanation \\
\hline 1 & Equally preferred \\
3 & Moderately preferred \\
5 & Strongly preferred \\
7 & Very Strongly preferred \\
9 & Extremely preferred \\
$2,4,6,8$ & Intermediate values (compromises) \\
\hline
\end{tabular}

Saaty's matrix. Relative weights of the compared elements are the components of the (normalized) principal eigenvector of a consistent PCM [20]. An $n \times n$ PCM $\mathbf{P}$ is (fully) consistent if $p_{i j}=p_{i k} p_{k j}, \forall i, j, k \in\{1, \ldots, n\}$. A consistency index $C I \triangleq \frac{\lambda_{\max }-n}{n-1}$ measures the consistency level of a Saaty's matrix, where $\lambda_{\max }$ is the principal eigenvalue. For a fully consistent matrix, $\lambda_{\max }=n$. In general, the lower $C I$ the less the inconsistency. AHP compares $C I$ with a random index $(R I)$ representing the average consistency index of many $n \times n$ Saaty's matrices randomly generated. Whenever $\frac{C I}{R I} \geq 0.1$, preferences have to be reformulated. The weight of each alternative with respect to the goal (i.e., its ranking) is 
computed by multiplying the relative weight of the alternative with respect to each criterion by the relative weight of that criterion. So-obtained values (one for each criterion) are then summed. The highest-ranking alternative is finally selected.

2) Technique for Order of Preference by Similarity to Ideal Solution (TOPSIS): TOPSIS is an MCDM technique [21]. For an $n$-alternatives and $m$-criteria decision problem, TOPSIS requires an $n \times m$ decision matrix $\mathbf{H}=\left[h_{i j}\right]$, where $h_{i j}$ is the performance of alternative $i$ on criterion $j$, with $i \in\{1, \ldots, n\}$ and $j \in\{1, \ldots, m\}$. A vector $\boldsymbol{\omega}=\left(\omega_{1}, \ldots, \omega_{m}\right)$, where $\sum_{j=1}^{m} \omega_{j}=1$, is also required, with the weights of the criteria as components. To select the best alternative, TOPSIS first computes the normalized decision matrix $\mathbf{R}=\left[r_{i j}\right]=$ $h_{i j} / \sqrt{\sum_{i=1}^{n} h_{i j}^{2}}$ and the weighted normalized decision matrix $\mathbf{V}=\left[v_{i j}\right]=v_{i j}=\omega_{j} r_{i j}$. Then, the ideal best $(I B)$ and worst $(I W)$ solutions are found. Let $\Omega_{B}$ and $\Omega_{C}$ contain the indexes of benefit and cost criteria, respectively. Let $I B=\left(a_{1}^{+}, \ldots, a_{m}^{+}\right)$and let $I W=\left(a_{1}^{-}, \ldots, a_{m}^{-}\right)$, where $a_{j}^{+}=\max _{i} v_{i j}$ for $j \in \Omega_{B}$ or $a_{j}^{+}=\min _{i} v_{i j}$ for $j \in \Omega_{C}$, and $a_{j}^{-}=\min _{i} v_{i j}$ for $j \in \Omega_{B}$ or $a_{j}^{-}=\max _{i} v_{i j}$ for $j \in \Omega_{C}$. TOPSIS measures the Euclidean distance of each alternative from $I B$, i.e., $D_{i}^{+}=\sqrt{\sum_{j=1}^{m}\left(v_{i j}-a_{j}^{+}\right)^{2}}$, and $I W$, i.e., $D_{i}^{-}=\sqrt{\sum_{j=1}^{m}\left(v_{i j}-a_{j}^{-}\right)^{2}}$. Finally, TOPSIS computes the relative closeness coefficient of each alternative to $I B$ as $R C L_{i}^{+}=D_{i}^{-} /\left(D_{i}^{+}+D_{i}^{-}\right):$the higher $R C L_{i}^{+}$the better. The best alternative is $k=\arg \max _{i} R C L_{i}^{+}$.

\section{B. Multi-objective optimization}

A multi-objective optimization (MOO) problem, in the standard form, consists in minimizing $\mathbf{f}(\mathbf{x})=\left[f_{1}(\mathbf{x}), \ldots, f_{k}(\mathbf{x})\right]$ such that $\mathbf{x} \in \mathcal{X}$ and $\mathcal{X}=\left\{\mathbf{x} \in \mathbb{R}^{p}: g_{i}(\mathbf{x}) \leq 0, h_{j}(\mathbf{x})=\right.$ $0, \forall i=1, \ldots, m, \forall j=1, \ldots, n\}$, where $k \geq 2$ is the number of objectives, $m$ and $n$ are the number of inequality and equality constraints delimiting the feasible region $\mathcal{X}$, respectively. The vector-valued objective function $\mathbf{f}: \mathbb{R}^{p} \rightarrow \mathbb{R}^{k}$ contains the objective functions as elements.

Since MOO problems have generally no solution minimizing all the objective functions, Pareto dominance and Pareto optimality have been introduced. Consider two feasible solutions $\mathbf{x}^{\mathbf{1}}, \mathbf{x}^{\mathbf{2}}$ of an MOO problem, i.e., $\mathbf{x}^{\mathbf{1}}, \mathbf{x}^{2} \in \mathcal{X}$. Pareto dominance says that $\mathbf{x}^{\mathbf{1}}$ dominates $\mathbf{x}^{\mathbf{2}}$ if $f_{i}\left(\mathbf{x}^{\mathbf{1}}\right) \leq f_{i}\left(\mathbf{x}^{\mathbf{2}}\right) \forall i \in$ $\{1, \ldots, k\}$ and $\exists j \in\{1, \ldots, k\}: f_{j}\left(\mathbf{x}^{\mathbf{1}}\right)<f_{j}\left(\mathbf{x}^{2}\right)$. Pareto optimality is met when it is impossible to improve a feasible solution with respect to any objective, without degrading at least one of the remaining. Pareto-optimal solutions in the objective space form the Pareto front.

1) Genetic algorithms: A genetic algorithm (GA) is a heuristic optimization method based on biological evolution [22]. GAs efficiently deal with complex single- or multiobjective optimization problems. Solutions, i.e., individuals, are encoded by using bit strings, integer- or real-valued vectors, etc. Each individual is typically made of one chromosome composed of genes.

GAs start by randomly generating a set of candidate solutions to the problem, forming the initial population. Each individual's goodness is measured by using a fitness function: e.g., for a minimization problem, the lower the fitness, the better the individual. Individuals with good fitness are more likely to be selected for reproduction, which takes place by means of crossover and/or mutation operators. A new population (offspring) is created by replacing (part of) the individuals of the current population with the newly-generated ones. The process iterates until a terminating condition is met. The fittest individual of the last population, or throughout all populations, is generally chosen as optimal solution.

2) The NSGA-II algorithm: The Non-dominated Sorting Genetic Algorithm (NSGA-II) [23] is an efficient multiobjective genetic algorithm based on Pareto dominance. As Fig. 1 shows, starting from a randomly generated population $P_{0}$ of $n$ chromosomes, NSGA-II assigns a non-domination rank to each one: rank 1 for the best level, rank 2 for the next level, etc. To this aim, NSGA-II looks for non-

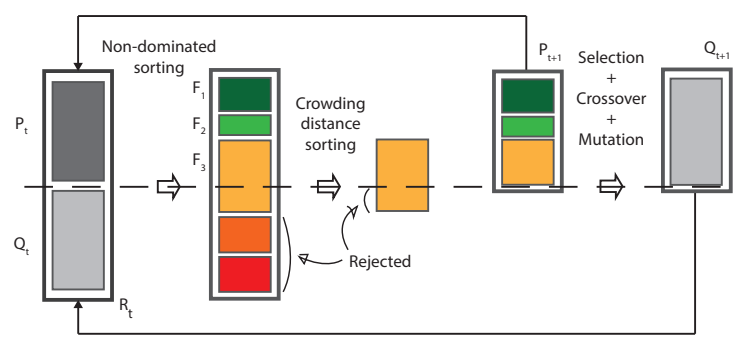

Fig. 1. The NSGA-II algorithm.

dominated chromosomes in $P_{0}$ and associates rank 1 with them: chromosomes with rank 1 form the first front. The chromosomes in the first front are then neglected to determine rank 2 chromosomes. The procedure is repeated until chromosomes are over. At iteration $t$, an offspring population $Q_{t}$ of $n$ individuals is generated by selecting from current population $P_{t}$, then performing crossover and mutation. A new population $R_{t}$ of $2 n$ chromosomes is generated merging $P_{t}$ and $Q_{t}$. Chromosomes in $R_{t}$ are assigned to their ranks, hence $R_{t}$ is partitioned into fronts. For each front, the density of individuals in each individual's neighborhood is estimated with the crowding distance, i.e., the sum of the distances from an individual to the closest one, along each objective. NSGA-II sorts individuals within each front using the crowding distance. Based on the sorting among fronts first, and then among individuals in the same front, the worst $n$ individuals are rejected. NSGA-II iterates until some stop condition is met.

\section{WORKER'S RISK PERCEPTION AND CAUTION}

The worker's risk perception and caution are modeled. A classification, implemented by the neural module, is then described to assign a worker to his/her risk perception and caution levels with respect to every task.

\section{A. Model}

Consider a workplace with a set of tasks $\mathcal{T}=\left\{t_{1}, \ldots, t_{|\mathcal{T}|}\right\}$ and let $\mathcal{W}=\left\{w_{1}, \ldots, w_{|\mathcal{W}|}\right\}$ be a set of workers. Task $t_{i} \in \mathcal{T}$ exposes a worker to a set $\mathcal{R}_{i}$ of risks. All the risks of the workplace are contained in set $\mathcal{R}=\bigcup_{i=1}^{|\mathcal{T}|} \mathcal{R}_{i}$. 
Each risk $r_{k} \in \mathcal{R}$ can be prevented by a set $\mathcal{A}_{k}=$ $\left\{a_{k, 1}, \ldots, a_{k,\left|\mathcal{A}_{k}\right|}\right\}$ of preventive actions. Preventive actions can decrease the probability of the risk materializing and/or make less injurious the consequences on health, the so-called risk impact. A preventive action has a prevention level in $\mathcal{L}=\{1, \ldots, L\}$. The higher the prevention level the more an action can reduce the risk probability and/or the risk impact. Prevention levels are determined by experts in risk assessment.

Consider a set of human factors (or simply factors) $\mathcal{H}=$ $\left\{h_{1}, \ldots, h_{|\mathcal{H}|}\right\}$. Each human factor $h_{v}$ takes values in a domain $\mathcal{D}_{v}$. Set $\mathcal{H}$ is composed of two kinds of human factors: relating to the individual and relating to the job (i.e., the task). The former set consists of $P$ personal factors, the latter of $T$ task-related factors. For worker $w_{j} \in \mathcal{W}$, the risk perception personal level pers_perc ${ }_{j}$ depends on vector $\left(d_{1, j}, \ldots, d_{P, j}\right) \in \mathcal{D}_{1} \times \cdots \times \mathcal{D}_{P}$, where $d_{v, j} \in \mathcal{D}_{v}$ is the value of personal factor $h_{v}$ for worker $w_{j}$. Therefore, a function $\varphi_{P E R S O N A L}: \mathcal{D}_{1} \times \cdots \times \mathcal{D}_{P} \rightarrow[0,1]$ exists, such that $\left(d_{1, j}, \ldots, d_{P, j}\right) \mapsto \varphi_{P E R S O N A L}\left(d_{1, j}, \ldots, d_{P, j}\right)=$ pers_perc ${ }_{j}$.

The perception level task_per $c_{i, j}$ of $w_{j}$ for the risks of task $t_{i}$ depends on vector $\left(d_{P+1, j}, \ldots, d_{P+T, j}\right) \in \mathcal{D}_{P+1} \times$ $\cdots \times \mathcal{D}_{P+T}$. Here, $d_{v, j}$ is the value of task-related factor $h_{v}$ for worker $w_{j}$. Also, task_perc $c_{i, j}$ is dependent on the risk perception personal level pers_perc $c_{j}$ of $w_{j}$. As a consequence, there exists a function $\varphi_{T A S K}: \mathcal{D}_{P+1} \times \cdots \times \mathcal{D}_{P+T} \times$ $[0,1] \rightarrow[0,1]$ such that $\left(d_{P+1, j}, \ldots, d_{P+T, j}\right.$, pers_perc $\left.c_{j}\right) \mapsto$ $\varphi_{T A S K}\left(d_{P+1, j}, \ldots, d_{P+T, j}\right.$, pers_perc $\left.{ }_{j}\right)=$ task_perc $_{i, j}$.

For every single risk $r_{k}$ and worker $w_{j}$, the caution of $w_{j}$ for $r_{k}$ depends on the number of preventive actions $w_{j}$ performs to protect himself/herself from $r_{k}$, for each prevention level. These preventive actions form the behavior of $w_{j}$ toward $r_{k}$. Let $\mathcal{A}_{k, \ell=\bar{\ell}} \subseteq \mathcal{A}_{k}$ be the set of $\bar{\ell}$-level actions to prevent $r_{k}$ and let $\# \mathcal{A}_{k, \ell=\bar{\ell}, j}$ denote the count of $\bar{\ell}$-level actions performed by $w_{j}$ to prevent $r_{k}$. There exists a function $\rho_{k}:\left\{0, \ldots,\left|\mathcal{A}_{k, \ell=1}\right|\right\} \times \cdots \times\left\{0, \ldots,\left|\mathcal{A}_{k, \ell=L}\right|\right\} \rightarrow[0,1]$, such that $\left(\# \mathcal{A}_{k, \ell=1, j}, \ldots, \# \mathcal{A}_{k, \ell=L, j}\right) \mapsto \rho_{k}\left(\# \mathcal{A}_{k, \ell=1, j}\right.$, $\left.\ldots, \# \mathcal{A}_{k, \ell=L, j}\right)=$ risk_caution $_{k, j}$, for each $k=1, \ldots,|\mathcal{R}|$.

Finally, for each task $t_{i}$ and worker $w_{j}$, the caution level of $w_{j}$ for $t_{i}$ is dependent on risk_caution $k, j, \forall k: r_{k} \in \mathcal{R}_{i}$. A group of functions $\tau_{i}:[0,1]^{\left|\mathcal{R}_{i}\right|} \rightarrow[0,1]$, one for each $i=1, \ldots,|\mathcal{T}|$, such that $\bigcup_{r_{k} \in \mathcal{R}_{i}}$ risk_caution $_{k, j} \mapsto$ $\tau_{i}\left(\bigcup_{r_{k} \in \mathcal{R}_{i}}\right.$ risk_caution $\left._{k, j}\right)=$ task_caution $_{i, j}$, map a configuration of risk cautions into the task caution of $w_{j}$, for each task $t_{i}$ of the workplace.

Worker $w_{j}$ is therefore represented by the tuple

$$
\theta_{j}=\left\{\bigcup_{v=1}^{P+T} d_{v, j}, \bigcup_{k=1}^{|\mathcal{R}|} \bigcup_{\lambda=1}^{L} \# \mathcal{A}_{k, \ell=\lambda, j}\right\}
$$

where $\bigcup_{v=1}^{P+T} d_{v, j}$ are the values of each human factor and $\bigcup_{k=1}^{|\mathcal{R}|} \bigcup_{\lambda=1}^{L} \# \mathcal{A}_{k, \ell=\lambda, j}$ are the counts of preventive actions for each prevention level, toward each risk. Note that $v \in$ $\{1, \ldots, P\}$ denotes personal factors, while task-related factors are $v \in\{P+1, \ldots, P+T\}$.

\section{B. Classification system}

A classification system was proposed in [24] to compute task_perc $c_{i, j}$ and task_caution ${ }_{i, j}$, respectively, by means of two multi-layer perceptron neural networks (MLPs), given the tuple of features in Eq. (1). Neural networks were used for being able to learn complex nonlinear relationships between variables, from observed data.

Semi-supervised learning within a stage-based learning scheme was used. A supervised learning stage first trains the MLPs separately. Then, each MLP's performance is improved thanks to what the other learned previously. To this aim, unsupervised data are used to generate, through each MLP, the desired outputs from the other. This is possible because the two MLPs, which receive as inputs two different representations of the same person, should produce coherent outputs. Sogenerated training sets are used to refine the training of the two MLPs starting from the values assumed by the neural parameters at the end of the previous stage. Different neural architectures and parameters configurations were tried for the two MLPs, and the best ones were found by means of an evolutionary computation-based approach.

The classification system described so far is implemented by the neural module of the optimization system here proposed to obtain the safety level of assigning task $t_{i}$ to worker $w_{j}$.

\section{OPTIMIZATION PROBLEM}

This section first presents the multi-objective optimization problem solved by the evolutionary module to generate near Pareto-optimal assignments. The section ends describing how the best assignment is chosen by the decision module.

\section{A. Objective functions}

Let $x_{i j} \in\{0,1\}$ be a decision variable such that

$$
x_{i j}= \begin{cases}1 & \text { if task } t_{i} \text { is assigned to worker } w_{j} \\ 0 & \text { otherwise }\end{cases}
$$

where $i \in\{1, \ldots,|\mathcal{T}|\}$ and $j \in\{1, \ldots,|\mathcal{W}|\}$. Vector $\mathbf{x} \in$ $\{0,1\}^{|\mathcal{T}| \times|\mathcal{W}|}$ represents a personnel assignment and contains variables $x_{i j}$ in lexicographic order. The objective functions, i.e., economic cost, expertise and safety, are rigorously defined in the following.

1) Economic cost: The cost of assigning task $t_{i}$ to worker $w_{j}$ stems from the sum of employment cost (i.e., what the employer pays for salary and benefits) and cost for the training. The cost for the training is as much higher as less experienced the worker is for the task assigned. Global cost is therefore:

$$
\operatorname{COST}(\mathbf{x})=\sum_{i=1}^{|\mathcal{T}|} \sum_{j=1}^{|\mathcal{W}|}\left(c_{j}^{E M P L O Y M E N T}+c_{i j}^{T R A I N I N G}\right) x_{i j}
$$

where $c_{j}^{E M P L O Y M E N T}$ is the employment cost of worker $w_{j}$ and $c_{i j}^{T R A I N I N G}$ is the cost for the training of $w_{j}$ if assigned to $t_{i}$. In the system proposed, global cost is minimized.

2) Expertise: Let $\mathcal{N}_{j}$ be the set of the past jobs of worker $w_{j}$ and let $\mathcal{N}_{i, j} \subseteq \mathcal{N}_{j}$ contain the past jobs where $w_{j}$ performed task $t_{i}$ or a task requiring similar skills. Also, let $D_{j, n}^{S T A R T}$ and $D_{j, n}^{E N D}$ be the dates on which $w_{j}$ began and ceased past job $n \in \mathcal{N}_{j}$. Finally, let ability . $_{i, j} \in[0,1]$ measure the skill of $w_{j}$ in executing $t_{i}$ based on how rapidly $w_{j}$ completes the task and with what quality level. Ability 
is typically assessed by industry experts. Higher values correspond to better skill. The experience ${ }_{i, j} \in[0,1]$ of worker $w_{j}$ for task $t_{i}$ is here modeled as the ratio of two terms: i) the count of the days $w_{j}$ has performed $t_{i}$ in life multiplied by his/her ability for that task; ii) the work seniority of $w_{j}$ measured from the date on which $w_{j}$ started his/her first job to the date on which $w_{j}$ ceased his/her last job. This second date may be the current date, if the last job is the current job of $w_{j}$. Formally, the experience of worker $w_{j}$ for task $t_{i}$ is

$$
\text { experience }_{i, j}=\frac{\operatorname{ability}_{i, j} \cdot \sum_{n \in \mathcal{N}_{i, j}} \operatorname{days}\left(D_{j, n}^{S T A R T}, D_{j, n}^{E N D}\right)}{\operatorname{days}\left(\min _{n \in \mathcal{N}_{j}} D_{j, n}^{S T A R T}, \max _{n \in \mathcal{N}_{j}} D_{j, n}^{E N D}\right)},
$$

where days () returns the time period (in days) delimited by the dates passed as arguments. The minimum/maximum date corresponds to the least/most recent date. Consider a task $t_{i}$ and two workers $w_{1}$ and $w_{2}$. If ability $_{i, 1}>$ ability $_{i, 2}$ then $w_{1}$ is more experienced according to Eq. (4) because $w_{1}$ has generally spent more time in performing $t_{i}$ in his/her working life than $w_{2}$. When ability $_{i, 1}=$ ability $_{i, 2}$, Eq. (4) assigns a greater experience to the worker who is more used to performing $t_{i}$. A worker is thought to be the more used to performing a task the longer he/she has performed that task during his/her working life. How used a worker is to performing a task is measured in Eq. (4) by the ratio $\sum_{n \in \mathcal{N}_{i, j}} \operatorname{days}\left(D_{j, n}^{S T A R T}, D_{j, n}^{E N D}\right) / \operatorname{days}\left(\min _{n \in \mathcal{N}_{j}} D_{j, n}^{S T A R T}\right.$ $\left.\max _{n \in \mathcal{N}_{j}} D_{j, n}^{E N D}\right)$. Finally, note that Eq. (4) assumes that workers have a minimum experience in manufacturing companies. Workers who just start their working life are highly likely to get hurt and are typically neglected in recruitment processes involving dangerous tasks until they get a minimum experience, e.g., by performing less dangerous tasks where similar skills are required. The global expertise of assignment $\mathbf{x}$ is defined as:

$$
\begin{aligned}
& \operatorname{EXPERTISE}(\mathbf{x})= \\
& -\sqrt{\sum_{i=1}^{|\mathcal{T}|} \sum_{j=1}^{|\mathcal{W}|}\left(\text { experience }_{i, j} x_{i j}-\max _{\substack{i, j \\
x_{i j}=1}} \text { experience }_{i, j}\right)^{2}}
\end{aligned}
$$

Global expertise is maximized, thus guaranteeing job assignments where workers are skilled enough for the task assigned.

3) Safety: Global safety is here thought of as the extent to which riskier tasks are assigned to workers with higher prudence, and vice versa. Given worker $w_{j}$ and task $t_{i}$, let

$$
\text { safety }_{i, j}=\omega_{H} \cdot \text { task_perc } c_{i, j}+\omega_{B} \cdot \text { task_caution }_{i, j}
$$

express how safely $w_{j}$ performs $t_{i}$, where task_per $c_{i, j}$ and task_caution $_{i, j}$ are obtained by means of the neural module described in detail in Section $\mathbf{V}$, and $\omega_{H}, \omega_{B} \in[0,1]$ such that $\omega_{H}+\omega_{B}=1$ are weights, specified by an expert, defining the importance of human factors and behavior, respectively. The global safety of assignment $\mathbf{x}$ is here defined as:

$$
\begin{aligned}
& S \operatorname{AFETY}(\mathbf{x})= \\
& -\sqrt{\sum_{i=1}^{|\mathcal{T}|} \sum_{j=1}^{|\mathcal{W}|}\left(\text { safety }_{i, j} x_{i j}-\max _{\substack{i, j \\
x_{i j}=1}} \text { safety }_{i, j}\right)^{2}} .
\end{aligned}
$$

Global safety is maximized by the system proposed in this paper so as to find job assignments $\mathbf{x}$ characterized by a good level of safety for every worker.

\section{B. Problem formulation}

Consider a set of tasks $\mathcal{T}$ and a set of workers $\mathcal{W}$ where $|\mathcal{W}| \lesseqgtr|\mathcal{T}|$, i.e., the number of workers may be either lower or greater than the number of tasks, or there may be as many workers as tasks. The optimization problem is:

$$
\begin{gathered}
\underset{\mathbf{x}}{\operatorname{Maximize}} \mathbf{f}(\mathbf{x})= \\
{[-\operatorname{COST}(\mathbf{x}), \operatorname{EXPERTISE}(\mathbf{x}), \operatorname{SAFETY}(\mathbf{x})]}
\end{gathered}
$$

subject to:

$$
\left\{\begin{array}{c}
\sum_{\substack{|\mathcal{T}| \\
|\mathcal{T}|}}^{\mid \mathcal{T}} x_{i j}=1, \quad \forall j=1, \ldots,|\mathcal{W}| \quad \text { reassignment } \\
\sum_{i=1}^{|\mathcal{W}|} x_{i j} \leq 1, \quad \forall j=1, \ldots,|\mathcal{W}| \quad \text { recruitment } \\
\sum_{j=1}^{\mid} x_{i j}=1, \quad \forall i=1, \ldots,|\mathcal{T}|
\end{array}\right.
$$

$$
\begin{gathered}
\text { experience }_{i j} x_{i j} \geq \text { experience }_{i}^{M I N} \quad \forall i=1, \ldots,|\mathcal{T}|, \\
\eta_{i} \geq \eta^{C R I T I C A L}, \forall j=1, \ldots,|\mathcal{W}| \\
x_{i j} \in\{0,1\}, \quad \forall i=1, \ldots,|\mathcal{T}|, \forall j=1, \ldots,|\mathcal{W}| .
\end{gathered}
$$

Equation $8 \mathrm{a}$ is the vector-valued objective function $\mathbf{f}(\mathbf{x})$ : $\{0,1\}^{|\mathcal{T}| \times|\mathcal{W}|} \rightarrow \mathbb{R}_{-}^{3}$ whose components are, in the order, the opposite of the global cost, the global expertise, and the global safety of personnel assignment $\mathbf{x} \in\{0,1\}^{|\mathcal{T}| \times|\mathcal{W}|}$. Constraints (8b), one for each worker, depend on the problem dealt with: for personnel reassignment, they let each worker be assigned to one task; for personnel recruitment, they consider a worker may be assigned to no task. Constraints (8c) let each task be assigned to one worker. Constraints (8d) establish a minimum experience experience ${ }_{i}^{M I N}$ to perform safety-critical tasks, i.e., $t_{i}$ with task hazardousness $\eta_{i}$ greater than a threshold $\eta^{C R I T I C A L} \in(0,1]$. Finally, Eq. $8 \mathrm{e}$ is the integer constraint.

\section{Detailed DESCRIPTION OF THE SYSTEM}

The optimization system proposed in this paper is shown in Fig. 2 and consists of three modules: neural module, evolutionary module and decision module. 


\section{A. Neural module}

For each worker $w_{j}$ and each task $t_{i}$, the neural module computes the two components (i.e., task_perc $c_{i, j}$ and task_caution $_{i, j}$ ) for determining the worker's level of safety safety $_{i, j}$ in interacting with the task, thanks to his/her human factors and behavior. From an architectural point of view, the neural module consists of two sub-modules, denoted in Fig. 2 with $A$ and $B$, respectively.

Still considering worker $w_{j}$ and task $t_{i}$, sub-module $\mathrm{A}$ receives the configuration of values of the worker's human factors (personal and task-related) as input. The values related to personal human factors (from $f_{1}$ to $f_{P}$ in Fig. 2) are the input to a multi-layer perceptron neural network, denoted with PPL (for "Personal Perception Level") in Fig. 2, which returns the risk perception personal level pers_perc $c_{j}$ of worker $w_{j}$. On the other hand, the values related to task-related human factors (from $f_{P+1}$ to $f_{P+T}$ in Fig. 2), together with the worker's risk perception personal level pers_perc $c_{j}$, form the input to another multi-layer perceptron neural network, indicated with RPT (for "Risk Perception for Task") in Fig. 2 . which returns $t a s k \_p e r c_{i, j}$, i.e., the level of risk perception of worker $w_{j}$ with respect to task $t_{i}$.

Sub-module B, still with reference to worker $w_{j}$ and task $t_{i}$, receives as inputs, for each level of prevention, the count of preventive actions that worker $w_{j}$ performs (or would perform) to deal with every single risk of task $t_{i}$. According to these inputs, sub-module B returns the caution task_caution ${ }_{i, j}$ of worker $w_{j}$ in dealing with task $t_{i}$. More precisely, for each risk $r_{k}$ of task $t_{i}$, a multi-layer perceptron neural network (denoted as $R_{k}$ in Fig. 2) receives in input, for each level of prevention, the count of preventive actions used by the worker to interact with risk $r_{k}$ and returns the level of prudence risk_caution ${ }_{k, j}$ of worker $w_{j}$ toward risk $r_{k}$. The values of risk_caution $k, j$, one for each risk $r_{k}$ of task $t_{i}$, are the input to a multi-layer perceptron neural network, denoted with CT (for "Caution for Task") in Fig. 2, which returns the level of task_caution ${ }_{i, j}$ of worker $w_{j}$ with respect to task $t_{i}$. Architectural details on the neural module can be found in [24], and are omitted here because they do not represent the main focus of the paper.

Given worker $w_{j}$ and a task $t_{i}$, the values of risk perception for task and caution for task are then used by the $S$ module (located downstream of the neural module, as one can see from Fig. 2 in order to compute safety $y_{i, j}$, according to Eq. (6). As stated before, safety $y_{i, j}$ measures the safety level of $w_{j}$ in performing task $t_{i}$ and, as a consequence, the safety level of assigning him/her to that task.

\section{B. Evolutionary module}

The evolutionary module is based on the NSGA-II algorithm and is aimed at generating $p$ near Pareto-optimal job assignments. Considered a set of tasks and a set of workers, the evolutionary module requires the following parameters as input, as shown in Fig. 2, i) all workers' safety levels in performing all tasks; ii) the cost resulting from assigning each worker to every single task; iii) the experience of each worker with respect to each task; iv) the safety-critical tasks identifiers. Values from i) to iii) let the evolutionary module compute global safety, cost and expertise (i.e., the objectives) of each job assignment, respectively. Values iv) serve as constraint in the problem.

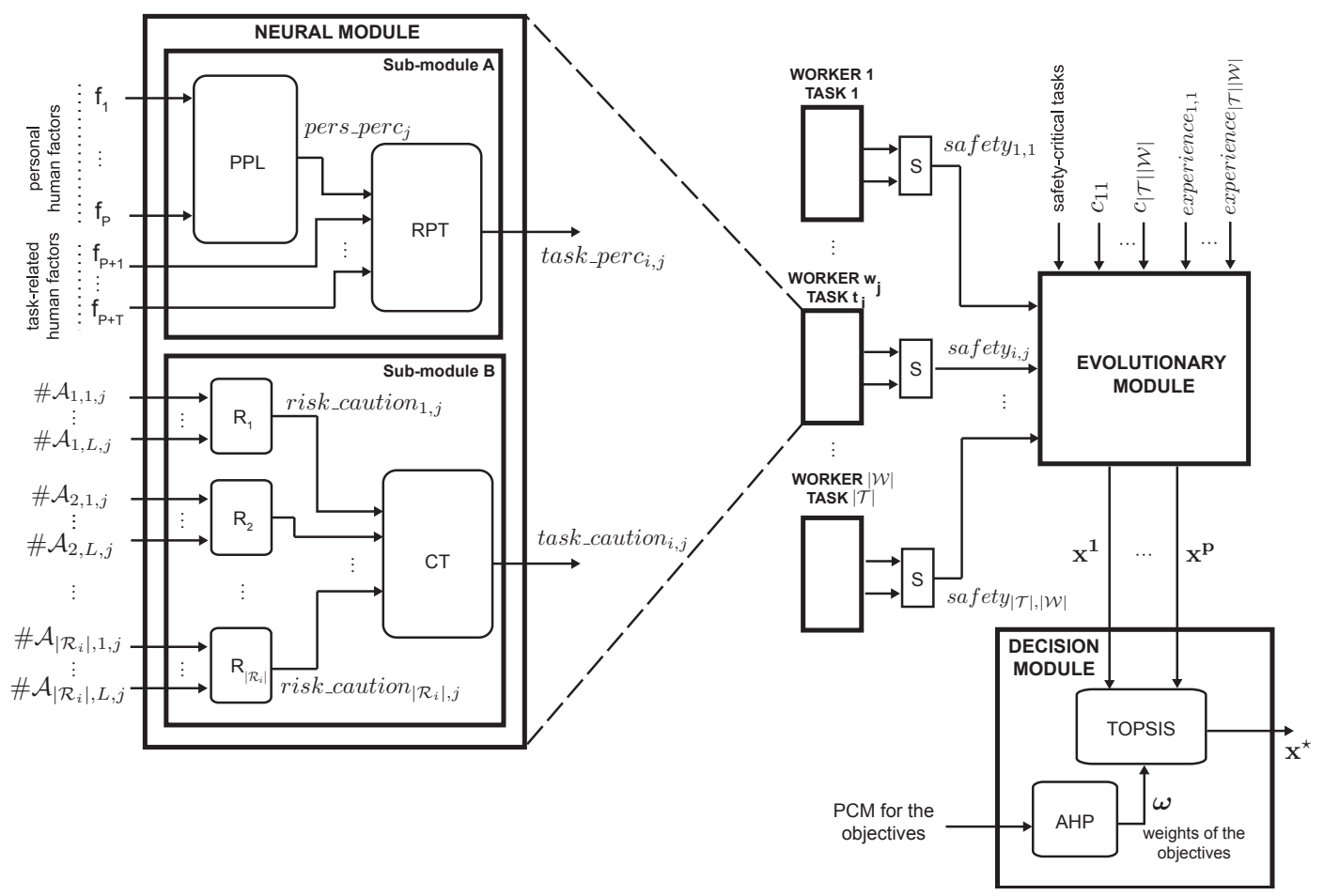

Fig. 2. Block diagram of the system. For the sake of simplicity, the cost of assigning worker $w_{j}$ to task $t_{i}$ is indicated just as $c_{i j}$, instead of $\left(c_{j}^{E M P L O Y M E N T}+c_{i j}^{T R A I N I N G}\right)$. Note that the neural module is replicated in the figure just to show that the same neural model (MLP) is used, with different inputs, to compute task_perc $i, j$ and $t a s k \_c a u t i o n_{i, j}$ for each $($ worker, task) pair. 
The evolutionary module also requires the parameters of the NSGA-II algorithm, chosen as specified in Section VI-D These parameters are neglected in Fig. 2 since they do not refer to the problem semantics.

\section{Decision module}

The decision module is composed of two sub-modules, indicated in Fig. 2 with AHP and TOPSIS, respectively. The decision module receives as input a pairwise comparison matrix (PCM) containing preferences related to the objectives, expressed by the management of the company in terms of the Saaty's scale. Also, the decision module takes the set of near Pareto-optimal job assignments generated by the evolutionary module as input. More in detail, the pairwise comparison matrix is the input to the AHP sub-module, which computes the weights of the objectives. The TOPSIS sub-module receives as input these weights and the set of near Pareto-optimal job assignments returned by the evolutionary module. As output, the TOPSIS module returns the near Pareto-optimal job assignment $\mathrm{X}^{\star}$ representing the best compromise of the decision maker's preferences. The output of the decision module is the output of the TOPSIS sub-module (see Fig. 2).

\section{DESCRIPTION OF THE EXPERIMENTS}

The proposed optimization system was tested on two realworld job assignment problems: personnel recruitment and personnel reassignment. Experiments were carried out involving two footwear companies.

\section{A. Dataset and implementation}

The optimization system was implemented in Java and MATLAB. Also, a website hosting a multiple choice test was implemented in Java EE and deployed on a web server. Data were saved into a MySQL database purposely developed. Workers (or candidates) of the factories involved in the experiments filled out the test, letting us acquire the data needed for the experiments anonymously.

Data gathering was hard due to privacy laws and because workers had to spend part of their working day in filling out the test. The dataset consists of 120 interviews. The footwear industry was chosen because of its serious risks.

For each worker $w_{j}$, the questionnaire collects:

- data related to personal factors, to compute pers_perc $c_{j}$;

- data about each past job, i.e., task involved, starting date and ceasing date (if exists), to compute experience $i, j$ for each task $t_{i}$

- data related to the behavior towards each risk $r_{k}$ of every single task $t_{i}$, i.e., the preventive actions selected by the worker from a proposed set. These selected actions are then partitioned depending on their prevention levels to compute task_caution ${ }_{i, j}$ for each task $t_{i}$.

For reasons of space, the questionnaire cannot be reported here. Anyway, with reference to the data related to the behavior, consider the cut risk as an example. The preventive actions used to characterize the worker's behavior are:

- activate the machinery safety elements;
- check the efficiency of the safety elements;

- put the gauntlet on;

- keep hands away from cutting elements;

- switch off the machine to fix faults;

- check and sharpen the cutting utensils periodically;

- no particular action.

Workers exposed to the cut risk are required to choose from the previous list of actions, each one associated with a prevention level. Note that, in the case of personnel recruitment, candidates may choose preventive actions artfully to appear more careful than they actually are. However, candidates are warned before the test of being responsible for the truthfulness of the answers. Also, candidates who pass the selection are subjected to a trial work period (typically a few days). In that period, they undergo risk-free simulations of the tasks to which they applied. Discrepancies between the answers the candidates gave in the questionnaire and their actual behavior entails immediate rejection.

Finally, to obtain how experienced candidates/workers are for each task, i.e., the values of experience $_{i, j}$ in Fig. 2, candidates/workers face risk-free practical tests. More precisely, considered worker $w_{j}$ and task $t_{i}$, the value of experience $e_{i, j}$ is an input to both the neural module and the evolutionary module. In the former case, it is a task-related factor (indicated with $f_{P+1}$ in Fig. 2 and is used to derive $t a s k \_p e r c_{i, j}$, which is then aggregated with task_caution $_{i, j}$ by Eq. (6), to obtain safety $_{i, j}$. In the latter case, experience ${ }_{i, j}$ is used to express a constraint of the assignment problem, i.e., constraint (8d).

Among the 120 collected interviews, 100 interviews relate to a recruitment process in the first factory, and were used to test the proposed framework on a real personnel recruitment problem (REC). Results are discussed in Section VII-A The remaining 20 interviews relate to the other factory wherein personnel reassignment (REA) was experimented. The results of this second experiment are discussed in Section VII-B

It is important to point out that the safety improvements described in the discussion are intended as a lower probability of the risk materializing and/or less harmful consequences of the risk. Of course, as it might be argued, in order to quantify the achieved improvement in safety, a factory where personnel recruitment/reassignment is performed with the help of the proposed system should be monitored for a statistically relevant period, so as to compare the number, as well as the severity, of the accidents happened before and after the implementation of the worker-task assignments produced by the optimization system described in this paper. Due to the aforementioned serious difficulties in data gathering, which mainly stem from the restrictiveness of the privacy laws, a statistical study of the accidents could not be made.

However, by maximizing the safety objective function in Eq. (7), each task is assigned to the person having the most suitable psycho-behavioral profile to perform the task with the highest level of safety achievable. Thus, considering the interaction with the risk by all the candidates of a personnel recruitment process, or, for the other problem, all the employees of a factory wherein personnel reassignment is performed, it follows that either the probability of the risk materializing or the impact of the risk (or both) can be decreased. 


\section{B. The shoe making process}

The shoe making process begins with the cutting of the leather by using knives and cutting machines, so as to prepare uppers, linings, reinforcements and insoles. Soles, heels, welts, vamps and accessories are obtained by using die cutters. While preparing the upper, the leather thickness is made uniform with milling cutters, then ornaments are added. The next step is stitching, where the upper is assembled by sewing all the parts. Folds are removed by pounding. By using lasting machines, the upper is then mounted on a last (a sculpture of the final shoe). The insole is joined to the upper, and prepared for the application of the sole by using a sanding machine. The sole is thus fixed to the upper by glueing, sewing or welts. The heel is fixed by nails or die casting. Heels may be coated with leather or wrapped with the same material of the upper. In the case of rubber soles, a press is used to fix the upper to a block made of sole and heel. The process continues with the finishing of the bottom of the shoe by: i) sanding heel and sole using rotating machines; ii) coloring and waxing the sole contour by using machines with rotating tools; iii) polishing heel and sole. Embellishment operations conclude the process: waxing-up the sole, cleaning the upper with solvents and/or brushes, starching and polishing. The shoes are finally put in a shoebox and stocked into the warehouse.

\section{Genetic encoding and genetic operators}

An integer-valued encoding is used by the evolutionary module. Chromosomes have as many genes as tasks. Each gene contains the identifier of the worker assigned to the task represented by that gene. Partially Matched Crossover [25] and swap mutation are used. With these genetic operators feasibility is maintained during evolution.

\section{Statistical validation of the NSGA-II parameters}

The best values of the parameters of the NSGA-II algorithm, i.e., crossover probability, mutation probability and number of chromosomes, were determined through simulations. More precisely, three values for each parameter were heuristically selected beforehand. These values are summarized in Table $\amalg$

TABLE II

PARAMETERS USED TO FIND THE BEST PARAMETRIZATION OF NSGA-II

\begin{tabular}{cc}
\hline Crossover probability & $\{0.25,0.55,0.85\}$ \\
Mutation probability & $\{0.01,0.05,0.09\}$ \\
Individuals & $\{250,300,350\}$ \\
\hline
\end{tabular}

Values in Table II were combined, thus obtaining 27 parameters configurations to be tested. The maximum number of iterations was kept to 1000. For each configuration, 20 runs were executed starting from different populations randomly generated. The best solution was selected by the decision module through TOPSIS, using weights determined by AHP, as each run of the evolutionary module terminated. The average global cost, expertise and safety of all the runs of the considered configuration were then computed. Statistical validation was carried out by means of the Student's $t$-test with 95\% confidence. Null hypothesis $\mathcal{H}_{0}$ assumes the difference on the mean values as dependent on chance. To calculate the Student's $t$-test values for each objective, 339 comparisons (i.e., $\left.\frac{(27-1)^{2}}{2}+1\right)$ were made. The configuration with the largest number of $\mathcal{H}_{0}$ rejections was selected.

\section{RESULTS AND DISCUSSION}

The discussion compares the worker-task assignment proposed by our system to the one suggested (or currently used) by the management of the involved factories. In the literature, to the best of the authors' knowledge, there is no previous system considering cost, worker's expertise and safety, as objectives, in job assignment scenarios. That is why no comparison with similar approaches can be made here. Data on the optimization time are neglected since no strict time constraint exists.

Because of the complexity of the shoe making process described in Section VI-B, the authors chose to base the discussion on the most and least dangerous tasks of a modern shoe factory. This is considered by the authors as a way to easily understand the contribution of the proposed system. The considered tasks, and, for each of them, machines, dangerous elements, utensils and risks involved, are in Table III

The discussion will show that our system makes the most of the workers' sensitivity to risk, by assigning them to the tasks that are suitable for their careful forethought. Firstly, in the recruitment scenario, it will be shown that dangerous tasks are assigned to cautious workers, while less dangerous tasks are assigned to scarcely prudent workers. This does not happen with the recruitment plan of the management. Secondly, the discussion of personnel reassignment will show that some highly prudent workers are currently assigned to trivial tasks: their ability to prevent risks is not considered at all. Also, several workers with high risk-taking are assigned to dangerous tasks just for being more experienced than others. With the proposed reassignment of workers to tasks, issues like these are overcome.

\section{A. Personnel recruitment (REC)}

1) Description of the problem: Personnel recruitment is faced by companies to select new employees for vacant posts. Experts generally determine how skilled candidates are in performing a task thanks to their past jobs and ability, through risk-free practical tests. In the considered company, candidates: i) provide a curriculum vitae; ii) perform some practical tests; iii) face an interview. Based on the outcome, each candidate is associated with a suitability level to each task he/she applies for. Cost is also taken into account. Assigning a candidate to a task is much more expensive the higher the candidate's work seniority and the lower his/her experience for that task.

Serious risks characterize the footwear industry, e.g., crush, amputation and fall. Notwithstanding, risk management is often carried out in SMEs just by assigning more dangerous tasks to more experienced workers. This is dangerous because more experienced workers tend to get higher illusion of control and this may reduce risk perception [11]. 
TABLE III

MOST (MD) AND LEAST (LD) DANGEROUS TASKS CONSIDERED IN THE DISCUSSION

\begin{tabular}{|c|c|c|c|c|c|}
\hline Task & Machines & Elements & Utensils & Risks & Type \\
\hline sole cutting & die cutters & blades & knives, scissors & $\begin{array}{l}\text { amputation, excoriations, crush, cut, fall, } \\
\text { scratches }\end{array}$ & MD \\
\hline milling & milling cutter & rotating rollers & - & $\begin{array}{l}\text { amputation, crush, dislocation, dragging, } \\
\text { entanglement }\end{array}$ & MD \\
\hline pressing & press & press & - & crush, fractures, nail loss & MD \\
\hline pounding & pounding machine & pounding disks & hammer & crush, fractures, lacerations & MD \\
\hline buffing & buffing machine & rotating brushes & - & abrasions, entanglement, excoriations & MD \\
\hline sanding & sanding machine & rotating abrasive belts & & cut, entanglement, grazes & MD \\
\hline ironing & iron & hot surfaces & Bunsen burners & burn, heat stress, scald & MD \\
\hline warehousing & forklift, strapping machine & - & $\begin{array}{l}\text { scissors, ladders, } \\
\text { ropes, transpallets }\end{array}$ & $\begin{array}{l}\text { fall, fall from heights, fall from stairs, muscle strain, } \\
\text { repeated environmental temperature changes }\end{array}$ & MD \\
\hline quality control & $-\quad . \quad 1$. & - & 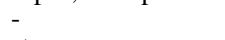 & eyestrain, posture pains & LD \\
\hline packaging & stamping machine & - & stamps & posture pains & LD \\
\hline
\end{tabular}

TABLE IV

WEIGHTS OF THE OBJECTIVES FOR PERSONNEL RECRUITMENT (REC) AND PERSONNEL REASSIGNMENT (REA) PROBLEMS

\begin{tabular}{lccc} 
& COST & EXPERTISE & SAFETY \\
\hline REC & 0.2 & 0.45 & 0.35 \\
REA & 0.35 & 0.25 & 0.4 \\
\hline
\end{tabular}

TABLE V

PARAMETERS USED FOR PERSONNEL RECRUITMENT (REC) AND PERSONNEL REASSIGNMENT (REA) PROBLEMS

\begin{tabular}{ccc} 
& REC & REA \\
\hline Chromosome encoding & \multicolumn{2}{c}{ Integer string } \\
Selection method & Binary tournament \\
Crossover operator & Partially matched crossover \\
Mutation operator & \multicolumn{2}{c}{ Swap mutation } \\
Population size & 300 & 250 \\
Crossover rate & 0.85 & 0.55 \\
Mutation rate & 0.05 & 0.01 \\
Max number of iterations & \multicolumn{2}{c}{1000} \\
\hline
\end{tabular}

2) Proposed technique for personnel recruitment: The optimization system was tested on a personnel recruitment process (REC) carried out by a footwear company to recruit 10 workers. The management gave 100 people the opportunity to apply. The management was first asked to fill a Saaty's matrix to compare the objectives. The weights of the objectives were derived by the decision module through AHP, and are shown in the first row of Table IV] An expert in risk assessment classified the preventive actions into three prevention levels (i.e., low, medium, high). Practical tests were required just for the post(s) each candidate applied for.

Data related to each candidate's human factors and behavior were gathered through the questionnaire described in Section VI-A Such data let the neural module obtain each candidate's task_perc and task_caution for each task. The safety of each candidate with respect to each task was then computed according to Eq. 6). The Pareto front (see Fig. 3. was approximated by using the parameters in Table $\mathrm{V}$.

The best solution (selected by the decision module) and the related values of the objectives are in Table VI In the table, the rows labeled with "Suggested" and "Current" contain, respectively, the personnel assignment suggested by the management for the recruitment scenario (REC), and currently used for the reassignment scenario (REA). The remaining rows, labeled with "Proposed", contain the assignment obtained by our system. Within each row, the cell under each task contains the identifier of the worker (or candidate) assigned to that task.

3) Discussion: The company needed 10 new workers for 4 vacant tasks, namely, packaging (3 workers), sole cutting ( 2 workers), glueing ( 4 workers) and sanding (1 worker). The aim here is to improve safety with low decrease in expertise. This stems from the similar weights of expertise and safety in Table IV] Cost plays a less important role.

The management suggested a recruitment plan with global safety equal to 5.26 (see Table VI). The proposed recruitment guarantees $\sim 89 \%$ improvement in safety. Since safety is a dimensionless new measure, some intuitive reasons for this improvement are discussed. Data on the workers' behavior with respect to every single task are not reported because each task exposes on average to 5 risks, in the experimented scenarios. Each risk can be prevented, on average, by 4 actions per prevention level, and our system considers 3 prevention levels. Therefore, if we were to consider the behavior of every worker with respect to the assigned task, there would be up to 60 preventive actions per worker, on average, with a total of 1200 preventive actions: 600 related to the assignment of the management and 600 related to the assignment of our system. Thus, we based the discussion on intuitive considerations about the behaviors that deeply impact on safety, to show how risks can be made less likely and/or less damaging by using our optimization system.

An important result of the proposed solution is that just under $62 \%$ of workers deal with the assigned task with highlevel preventive actions only. This percentage includes the workers assigned to the most dangerous tasks. The most unsafe strategy of the proposed recruitment is made of one medium-level action and one low-level action. This happens for packaging, which is a task whose most hazardous risk may cause just temporary poor posture pains. Instead, in the recruitment plan of the management, two safety-critical tasks are assigned to workers with behavior made of only one lowlevel preventive action. These tasks are dangerous because they deal with cutting machines and belt conveyors; workers may get caught by belts and even dragged onto the sharp elements.

By comparing our recruitment plan to the one of the management considering the tasks of Table III, it is important to note that two of the three candidates assigned by the management to packaging (tasks 3,6 and 7, in Table VI) had also applied for sanding (task 2, in Table VI). With reference 
TABLE VI

PERSONNEL RECRUITMENTS AND REASSIGNMENTS WITH CORRESPONDING OBJECTIVES VALUES FOR THE EXPERIMENTED SCENARIOS

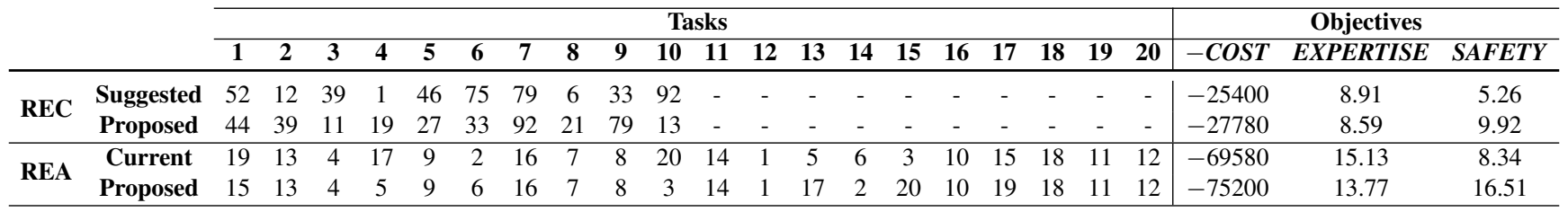

to packaging (a low-risk task), two of the three candidates assigned to it by the management, i.e., candidates 39 and 75, have a past experience of 4 and 6.5 years for the task, respectively (see Table VII). Even though they also applied for sanding and have levels of safety for it equal to 0.84 and 0.79 respectively, as shown in Table VII the management assigns sanding to candidate 12 , who has over 5 years of experience for that task, but a level of safety equal to 0.26 . As Table III shows, sanding exposes to serious risks. So the management does not make the most of how cautious candidates 39 and 75 actually are. As evidence, the proposed system assigns the more cautious candidate of the previous (i.e., 39) to sanding. Candidate 39 has just under 3 years of experience for sanding (see Table (VII), and this helps minimize the cost for the training, while maintaining good global expertise.

Sole cutting (tasks 9 and 10 in Table $\mathrm{VI}$ ) is assigned by the management to candidates 33 and 92 , who have more than 5 years of experience for that task, and ability higher than 0.8. Notwithstanding, they have a poor level of safety for sole cutting, which is equal to 0.22 and 0.31 , respectively. Also, candidate 92 had two accidents at work in the past, performing the same task. Sole cutting deals with blades and die cutters (see Table III), and further accidents might result in serious consequences. It is thus quite dangerous to recruit such candidates for sole cutting. As evidence, our system assigns both to packaging, as candidate 33 also applies for packaging, and candidate 92 also applies for packaging and warehousing. Packaging is chosen by the system probably because of its low complexity, which results in a low cost for the training. Also, packaging is an almost risk-free task, which is ideal for workers who may have become familiar with risk. These workers have low risk awareness, and this may result in dangerous behaviors during the task execution.

Finally, our recruitment maintains global expertise quite unaltered. Also, the improvement in safety is achieved at the expense of just $9.37 \%$ increase in global cost (see Table VI).
TABLE VII

INFORMATION ON THE DISCUSSED CANDIDATES

\begin{tabular}{|c|c|c|c|c|c|}
\hline \multirow{2}{*}{ Candidate } & \multicolumn{3}{|c|}{ Applications } & \multicolumn{2}{|c|}{ Assigned task } \\
\hline & Task & Experience & Safety & Management & System \\
\hline 39 & $\begin{array}{l}\text { packaging } \\
\text { sanding }\end{array}$ & $\begin{array}{c}4 y \\
2 y 8 m\end{array}$ & $\begin{array}{l}2.52 \\
0.84\end{array}$ & packaging & sanding \\
\hline 75 & $\begin{array}{l}\text { packaging } \\
\text { sanding }\end{array}$ & $\begin{array}{c}6 y=6 m \\
-\end{array}$ & $\begin{array}{l}1.68 \\
0.79\end{array}$ & packaging & - \\
\hline 12 & sanding & $5 y 6 \mathrm{~m}$ & 0.26 & sanding & - \\
\hline 33 & $\begin{array}{l}\text { sole cutting } \\
\text { packaging }\end{array}$ & $\begin{array}{c}5 y \\
2 m \\
-\end{array}$ & $\begin{array}{l}0.22 \\
2.84\end{array}$ & sole cutting & packaging \\
\hline 92 & $\begin{array}{l}\text { sole cutting } \\
\text { warehousing } \\
\text { packaging }\end{array}$ & $\begin{array}{c}5 y 9 m \\
- \\
8 m\end{array}$ & $\begin{array}{l}0.31 \\
0.45 \\
3.17\end{array}$ & sole cutting & packaging \\
\hline
\end{tabular}

\section{B. Personnel reassignment (REA)}

1) Description of the problem: Personnel reassignment aims to improve some aspect by reallocating personnel. Our optimization system was applied to a real-world scenario related to a footwear company, aimed at increasing safety with low penalization of cost and expertise. The next sub-sections contain details on the experiments and discuss the results.

2) Experiments on personnel reassignment: A PCM was first filled by the management to let the decision module obtain the priorities of the objectives. Data related to each worker's human factors and behaviors toward each risk were gathered thanks to the worker's answers to the questionnaire (see Section VI-A). Each worker's task_perc and task_caution were then determined by the neural module. The Pareto front was approximated using the parameters in Table $\mathrm{V}$

3) Motivation: The experiments aim to show how industrial safety can be increased (in terms of decrease in probability and/or impact of the risks) just by reassigning some tasks to more appropriate workers. Our optimization system may considerably help SMEs reallocate the personnel to increase safety, while maintaining reasonable costs. This is key because
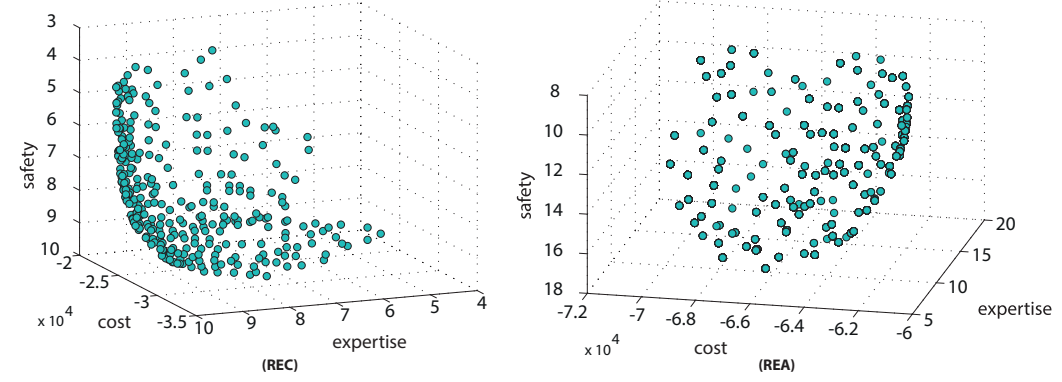

Fig. 3. Pareto fronts of REC and REA. Circles represent Pareto-optimal recruitments/reassignments. Costs are expressed in Euros. 
SMEs are usually small family realities with few employees and not sufficient funds for safety training.

4) Discussion: REA is related to 20 employees. Global cost and global safety are paramount. The management thus aims to get a low-cost safety improvement. Also, global expertise is not so marginal (see Table IV). The best solution achieved is in Table VI Our optimization system can highly increase safety by reassigning just 8 employees. Global safety is almost doubled at the expense of $\sim 8 \%$ increase in global cost. However, here the increase is just temporary as it stems from the cost for the training of the reassigned workers.

TABLE VIII

ABILITY AND PAST EXPERIENCE OF THE REASSIGNED WORKERS WITH RESPECT TO THE NEW TASK

\begin{tabular}{cccc}
\hline Worker ID & New task & Past experience [years] & Ability \\
\hline 2 & 14 & 0 & 0.75 \\
3 & 10 & 2.5 & 0.9 \\
5 & 4 & 0 & 0.7 \\
6 & 6 & 0 & 0.75 \\
15 & 1 & 0 & 0.8 \\
17 & 13 & 1 & 0.9 \\
19 & 17 & 0 & 0.85 \\
20 & 15 & 3 & 0.95 \\
\hline
\end{tabular}

To understand the improvement in safety, behaviors are first discussed. Then, the tasks in Table III are considered.

Behaviors made only of high-level preventive actions pass from 1 (current assignment) to 6 (proposed reassignment). Also, in the reassignment no worker has a behavior made only of low-level preventive actions for the assigned task. Instead, in the current assignment, 5 such behaviors exist. The riskiest ones concern two tasks (warehousing and milling) where workers may die falling from platforms and may experience severe grazes to the hands, respectively.

Reassigned workers perform 4 of the tasks of Table III ironing, packaging, milling and quality control. The task and worker identifiers below refer to Table VI

Ironing (task 10) is reassigned to an employee (worker 3) who currently performs quality control (task 15). The current assignment does not make the most of the risk sensitivity of worker 3 because he has a level of safety equal to 1.38 for ironing (see Table IX), which is more than three times greater than the level of safety of the employee who currently performs ironing (worker 20), which is equal to 0.41. Also, worker 20 is highly anxious (anxiety was assessed using the Zung test in the questionnaire) and this may promote accidents. Worker 20 has already been the victim of two accidents, with three working months of overall absence from work and a cost for the company just under 15,000 Euros. With reference to the expertise, one may reasonably assume that our system considers worker 3 as more suited for ironing rather than quality control (a low-risk task) because worker 3 has already performed the task for 2.5 years (see Table VIII). This guarantees a low cost for the training.

Regarding milling (tasks 13 and 14), Table IX] shows that the employees who currently carry it out (workers 5 and 6) have an average level of safety equal to 0.19 and a previous experience of $\sim 5.7$ years, on average. Also, worker 6 has recently been the victim of an accident where one of his fingers
TABLE IX

INFORMATION ON THE REASSIGNED WORKERS' CURRENT AND NEW POSITIONS

\begin{tabular}{cccccc}
\hline \multirow{2}{*}{ Worker } & \multicolumn{3}{c}{ Current position } & \multicolumn{2}{c}{ New position } \\
\cline { 2 - 6 } & Task & Experience & Safety & Task & Safety \\
\hline 3 & quality control & $7 \mathrm{~m}$ & 0.67 & ironing & 1.38 \\
20 & ironing & $1 \mathrm{y} 3 \mathrm{~m}$ & 0.41 & quality control & 1.52 \\
5 & milling & $5 \mathrm{y} 5 \mathrm{~m}$ & 0.16 & packaging & 1.55 \\
6 & milling & $5 \mathrm{y} 11 \mathrm{~m}$ & 0.22 & packaging & 1.69 \\
2 & packaging & $5 \mathrm{y}$ & 1.61 & milling & 2.9 \\
17 & pakaging & $5 \mathrm{y}$ & 1.83 & milling & 2.5 \\
\hline
\end{tabular}

has been crushed, with consequent amputation. On the other side, worker 5 says (among the preventive actions) to fix the machine by himself, and not to wear protective gloves so as to work faster. Although the employee's intention is to be more efficient, such measures put him at high risk of injury. Both employees are thus removed from milling and reallocated to packaging (tasks 4 and 6), a low-risk task in Table III. This can be reasonably considered as an effective precaution to avoid accidents at work. Milling is assigned to workers 2 and 17, who have been performing packaging for the last 5 years. As one can see from Table IX, both workers are moved to a more risky task (i.e., milling) because they have a high level of safety for that task, on average equal to 2.7 , versus the average level of safety of the workers who currently perform milling (workers 5 and 6), which is equal to 0.19 . The reason for such a poor level of safety might stem, e.g., from the correlation between the skill acquired in just under 6 years (see Table IX) and the illusion of control, i.e., the tendency for people to overestimate their ability to control events or situations. Some preventive actions that lead to the good level of safety for milling of the employees reassigned to that task (2 and 17) include: always wear reinforced gloves; stop working as soon as the machine fails; continuously monitor the efficiency of the safety devices; not fix a faulty machine without the intervention of the specialized staff. No action of these exist in the behavior of the employees currently assigned to milling (workers 5 and 6). About the expertise, only one of the employees reassigned to milling (i.e., worker 17) has previous experience for that task, amounting to one year (see Table VIII). However, the average ability of the employees reassigned to milling is 0.85 (see Table VIII). This ensures good skill and moderate financial resources required to train both workers, so as to meet the requirement on the cost, which is here the most important objective together with safety.

Finally, moving the focus on the least relevant objective, i.e., global expertise, Table VI shows that it passes from 15.13 to 13.77 , with $8.99 \%$ reduction. Five of the reassigned workers have never performed the newly assigned task in life. However, as it can be seen from the grey rows of Table VIII, they are characterized by an ability greater than (or equal to) 0.7 , which ensures fast learning of the new tasks. The remaining three workers have performed the newly assigned task previously, with periods ranging from 1 to 3 years. So, although global expertise was reduced by our system, one can reasonably expect that all workers will be sufficiently skilled to perform the tasks correctly and safely in the short term. 


\section{CONCLUSION}

This paper presented an integrated optimization system which helps the management of a company assign workers to tasks minimizing cost, and maximizing expertise and safety. The system is made of three modules. A neural module determines how safely a worker can be assigned to a task by using some worker's human factors and the accuracy of the preventive actions performed during the task execution. The multi-objective job assignment problem is solved by a hybrid evolutionary/MCDM resolution methodology. The evolutionary module generates an accurate approximation of the Pareto front by means of the NSGA-II algorithm. An MCDM technique based on AHP and TOPSIS is used by the decision module to select the near Pareto-optimal solution representing the best compromise of the preferences expressed by the management.

Experiments were carried out thanks to 120 interviews provided by workers (or candidates) of two manufacturing SMEs where personnel recruitment and personnel reassignment were carried out, respectively.

Compared to the solution suggested by the management, global safety in the workplace is more than doubled thanks to the proposed recruitment, with no reduction in expertise and very low increase in cost. Outstanding increase in safety was also achieved in the reassignment scenario by reallocating few workers. This entailed low increase in cost, which is paramount for SMEs.

The main novelty of this work consists in considering human factors and the worker's behavior to derive safety in personnel recruitment and reassignment scenarios. Every single task can thus be assigned to the person showing an optimal level of safety in interacting with it. By taking advantage of the workers' own attitude to prevent the risks of the task they perform, the proposed system can help make risks less likely and/or less injurious to save lives and get ever safer workplaces for years to come.

\section{REFERENCES}

[1] European Statistics on Accidents at Work (ESAW). Eurostat, 2013.

[2] Census of Fatal Occupational Injuries Summary. US. Bureau of Labor Statistics, 2014.

[3] http://www.ilo.org. International Labour Organization Statistics Database (ILOSTAT).

[4] Health and Safety Executive, Reducing error and influencing behaviour. HSE Books, 1999.

[5] M. Bouyer et al., "Personality correlates of risk perception," Risk Analysis, vol. 21, no. 3, pp. 457-465, 2001.

[6] B. Chauvin et al., "Risk perception and personality facets," Risk Analysis, vol. 27, no. 1, pp. 171-185, 2007.

[7] E. Peters and P. Slovic, "The role of affect and worldviews as orienting dispositions in the perception and acceptance of nuclear power," $J$. of Applied Social Psychology, vol. 26, pp. 1427-1428, 1996.

[8] L. Sjöberg, "Factors in risk perception," Risk Analysis, vol. 20, no. 1, 2000.

[9] P. Slovic, "Perception of risk," Science, vol. 236, no. 4799, pp. 280-285, 1987.

[10] M. S. Horswill and F. P. McKenna, "The effect of perceived control on risk taking," J. of Applied Social Psychology, vol. 29, no. 2, pp. 377-391, 1999.

[11] C. T. F. Klein and M. Helweg-Larsen, "Perceived control and the optimistic bias: a meta-analytic review," Psychology \& Health, vol. 17, no. 4, pp. 437-446, 2002

[12] J. Costa-Font et al., "Optimism and the perception of new risks," J. of risk research, vol. 12, no. 1, pp. 27-41, 2009.
[13] K.-Y. Teng et al., "Risk and safety program performance evaluation and business process modeling," IEEE Trans. Syst., Man, Cybern. A, Syst., Humans, vol. 42, no. 6, pp. 1504-1513, 2012.

[14] B. Lazzerini and F. Pistolesi, "Classifying workers into risk sensibility profiles: a neural network approach," in 8th European Modelling Symposium (EMS 2014), Pisa, IT, 2014, pp. 33-38.

[15] B. Lazzerini and F. Pistolesi, "Profiling risk sensibility through association rules," Expert Systems with Applications, vol. 40, no. 5, pp. 1484 1490, 2013.

[16] S. Bandini et al., "Case-based reasoning to support work and learning in small and medium enterprises," in 21st IEEE Int. Conf. on Tools with Artificial Intelligence (ICTAI 2009), Newark, NJ, USA, 2009, pp. 253-260.

[17] D. Dubois, "Representation, propagation, and decision issues in risk analysis under incomplete probabilistic information," Risk Analysis, vol. 30, no. 3, pp. 361-368, 2010.

[18] B. Lazzerini and L. Mkrtchyan, "Analyzing risk impact factors using extended fuzzy cognitive maps," IEEE Systems J., vol. 5, no. 2, pp. 288-297, june 2011.

[19] T. Saaty, "How to make a decision: The analytic hierarchy process," European J. of Operational Research, no. 48, pp. 9-26, 1990.

[20] T. L. Saaty, "Decision-making with the AHP: Why is the principal eigenvector necessary," European J. of Operational Research, vol. 145, pp. 85-91, 2003.

[21] K. Y. Ching-Lai Hwang, Multiple attribute decision making. SpringerVerlag, 1981.

[22] J. H. Holland, Adaptation in Natural and Artificial Systems: An Introductory Analysis with Applications to Biology, Control and Artificial Intelligence. Cambridge, MA, USA: MIT Press, 1992.

[23] K. Deb et al., "A fast and elitist multiobjective genetic algorithm: NSGAII," IEEE Trans. Evol. Comp, vol. 6, no. 2, pp. 182-197, 2002.

[24] M. Cococcioni et al., "A semi-supervised learning-aided evolutionary approach to occupational safety improvement," in IEEE Congress on Evolutionary Computation (CEC 2016), Vancouver, CA, 2016.

[25] D. E. Goldberg and R. Lingle, Jr., "Alleles loci and the traveling salesman problem," in 1st International Conference on Genetic Algorithms, Hillsdale, NJ, USA, 1985, pp. 154-159.

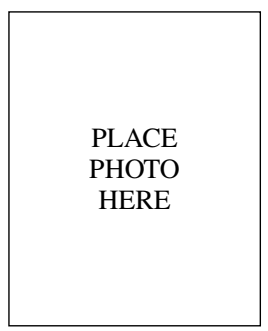

Beatrice Lazzerini (M'98) is a Full Professor of Computer Engineering with the Department of Information Engineering, University of Pisa, Italy. She has coauthored seven books and has published over 210 papers in international journals and conferences. She is coeditor of two books. She was involved and had roles of responsibility in several national and international research projects and scientific events. Her research interests are in the field of Computational Intelligence, with particular emphasis on fuzzy systems, neural networks and evolutionary computation, and their applications to pattern classification, risk analysis and management, diagnosis, forecasting, and multi-criteria decision making.

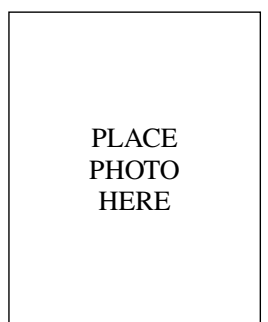

Francesco Pistolesi is a postdoctoral researcher with the Department of Information Engineering, University of Pisa, Italy. He graduated summa cum laude in Computer Engineering (curriculum Enterprise Systems) and received a $\mathrm{PhD}$ degree in Information Engineering (curriculum Computer System Architectures), both from the University of Pisa. His research interests embrace computational intelligence and data mining, with applications to decision support and multi-objective optimization. His current research activity is mainly focused on innovative solutions for smart industries, from industrial processes to occupational health and safety. 